\title{
Intratracheal transplantation of human umbilical cord blood-derived mesenchymal stem cells attenuates Escherichia coli-induced acute lung injury in mice
}

Eun Sun Kim ${ }^{1 \dagger}$, Yun Sil Chang ${ }^{1,2+}$, Soo Jin Choi ${ }^{3}$, Jin Kyu Kim ${ }^{1}$, Hey Soo Yoo ${ }^{1}$, So Yoon Ahn ${ }^{1}$, Dong Kyung Sung ${ }^{2}$, Soo Yoon $\mathrm{Kim}^{2}$, Ye Rim Park ${ }^{2}$ and Won Soon Park ${ }^{1,2^{*}}$

\begin{abstract}
Background: Human umbilical cord blood (UCB)-derived mesenchymal stem cells (MSCs) attenuate hyperoxic neonatal lung injury primarily through anti-inflammatory effects. We hypothesized that intratracheal transplantation of human UCB-derived MSCs could attenuate Escherichia coli (E. coli)-induced acute lung injury (ALI) in mice by suppressing the inflammatory response.

Methods: Eight-week-old male ICR mice were randomized to control or ALI groups. ALI was induced by intratracheal $E$. coli instillation. Three-hours after E. coli instillation, MSCs, fibroblasts or phosphate-buffered saline were intratracheally administered randomly and survival was analyzed for 7 days post-injury. Lung histology including injury scores, myeloperoxidase (MPO) activity, and protein levels of interleukin (IL)-1 $\alpha, I L-1 \beta, I L-6$, tumor necrosis factor (TNF)- $\alpha$, and macrophage inflammatory protein (MIP)-2 as well as the wet-dry lung ratio and bacterial counts from blood and bronchoalveolar lavage (BAL) were evaluated at 1, 3, and 7 days post-injury. Levels of inflammatory cytokines in the lung were also profiled using protein macroarrays at day 3 post-injury which showed peak inflammation.

Results: MSC transplantation increased survival and attenuated lung injuries in ALI mice, as evidenced by decreased injury scores on day 3 post-injury and reduced lung inflammation including increased MPO activity and protein levels of IL-1 $\alpha, I L-1 \beta, I L-6, T N F-\alpha$, and MIP-2 on day 3 and 7 post-injury. Inflammatory cytokine profiles in the lungs at day 3 post-injury were attenuated by MSC transplantation. MSCs also reduced the elevated lung water content at day 3 post-injury and bacterial counts in blood and BAL on day 7 post-injury.
\end{abstract}

Conclusions: Intratracheal transplantation of UCB-derived MSCs attenuates E. coli-induced ALI primarily by downmodulating the inflammatory process and enhancing bacterial clearance.

Keywords: Acute respiratory distress syndrome, Infection, Inflammation, Escherichia coli, Animal

\section{Background}

Acute respiratory distress syndrome (ARDS), a severe form of acute lung injury (ALI), is an acute respiratory failure in critically ill patients [1]. The mortality of ARDS/ALI remains unacceptably high because of the lack of effective treatments [2]. Infection is the most common cause of

\footnotetext{
* Correspondence: wonspark@skku.edu

+ Contributed equally

'Department of Pediatrics, Samsung Medical Center, Sungkyunkwan

University School of Medicine, Seoul, Korea

Full list of author information is available at the end of the article
}

ARDS/ALI and results in a higher mortality rate than noninfectious ALI [3,4]. The inflammatory process plays a key role in the pathogenesis of both infectious and noninfectious ALI, and the degree of acute inflammation is strongly correlated with outcome [5].

Recently, transplantation of various stem or progenitor cells such as bone marrow (BM)-derived mesenchymal stem cells (MSCs) or endothelial progenitor cells was reported to reduce mortality and attenuate ALI induced by endotoxins or sepsis in a rodent model [6-8]. This attenuation was associated with moderation of the

\section{C) Biomed Central}


inflammatory reactions that accompany ALI, with variable anti-bacterial effects $[9,10]$. These studies indicate that MSC treatment could be a new therapeutic modality for the treatment of ALI. Among the various sources of stem cells, human umbilical cord blood (UCB) provides readily available MSCs with low immunogenicity [11-13]. Therefore, human UCB-derived MSCs are regarded as a viable candidate source for cell therapy; however, this has yet to be studied in an in vivo ARDS/ALI model. We previously demonstrated that intratracheal transplantation of human umbilical cord blood (UCB)-derived MSCs attenuates hyperoxic lung injury in newborn rats through antiinflammatory effects rather than direct regeneration $[14,15]$.

We thus hypothesized that intratracheal transplantation of human UCB-derived MSCs could attenuate Escherichia $\operatorname{coli}$ (E. coli)-induced ALI in adult mice, and if so, the protective mechanism might be primarily mediated by antiinflammatory effects. In this study, we used a clinically relevant mouse model of infectious ARDS/ALI with Gram-negative bacterial pneumonia and sepsis induced by intratracheal instillation of E. coli [16]. We examined the effects of intratracheal delivery of human UCB-derived MSCs on survival, histology, and lung inflammation in mice with $E$. coli-induced ALI. Histological injury scores and myeloperoxidase (MPO) activity in lung homogenates were evaluated. Interleukin (IL)-1 $\alpha$, IL-1 $\beta$, IL-6, tumor necrosis factor (TNF)- $\alpha$, and macrophage inflammatory protein (MIP)-2 protein levels in lung homogenates were measured serially by ELISA. Mouse lungs showed peak inflammation three days after injury, at which time they were profiled using protein macroarray analysis. We chose this high-output proteomics approach for identifying proteins of interest in an effort to elucidate the potential mechanisms by which human UCB-derived MSCs modulate inflammatory responses. The wet-dry lung ratio and bacterial concentrations in bronchoalveolar lavage (BAL) and blood specimens after UCB-derived MSC transplantation were also examined.

\section{Methods}

\section{Cell preparation}

This study was approved by the Institutional Review Board of Samsung Medical Center and by Medipost, Co., Ltd, Seoul, Korea. UCB is the most promising source of MSCs because of its easy availability and the low immunogenicity of the cells; MSCs from UCB can be administered between HLA-incompatible individuals due to these cells' immune-modulatory properties, without alloreactive lymphocyte proliferative responses [11-13]. In the present study, MSCs were isolated and cultivated from human UCB as previously reported [14,15]. UCB was collected from umbilical veins after neonatal delivery with informed consent from pregnant mothers. The cells were shown to express CD105 and CD73 (99.6\% and $96.3 \%$, respectively) but not CD34, CD45, or CD14 (0.1\%, $0.2 \%$, and $0.1 \%$, respectively). The cells were positive for HLA-AB (96.8\%) but not HLA-DR (0.1\%). Human UCBderived MSCs differentiated into various cell types such as respiratory epithelium, osteoblasts, chondrocytes and adipocytes upon specific in vitro induction stimuli [15]. We confirmed the differentiation potential and karyotypic stability of the human UCB-derived MSCs up to the $11^{\text {th }}$ passage [15]. In this study, $5^{\text {th }}$ passage human UCBderived MSCs from a single donor were used for the transplantions.

Human fibroblasts (MRC-5; Korean Cell Line Bank No.10171) were obtained from the Korean Cell Line Bank (Seoul, Korea) and cultured in $\alpha$-MEM medium supplemented with $10 \%$ fetal bovine serum.

\section{Bacterial preparation}

We used E. coli as the source of infection because it is a common cause of gram-negative bacterial lung infection [16]. The E. coli strain E69 was generated by Pl transduction of the E. coli K12 outer membrane protein A (Omp A) gene into an Omp $\mathrm{A}^{-}$mutant of RS 218, isolated from the CSF of a newborn with E. coli meningitis (a gift from Dr. Kwang Sik Kim, Johns Hopkins Hospital, Baltimore, MD, USA) [17]. E. coli was cultured overnight in $10 \mathrm{~mL}$ of brain heart infusion broth (BHI, Difco Laboratories, Detroit, MI, USA) at $37^{\circ} \mathrm{C}$. The bacteria were then diluted in BHI media and grown for $1 \mathrm{~h}$ to mid-logarithmic phase. The suspension was centrifuged for $10 \mathrm{~min}$ at 5,000 $\mathrm{g}$ and washed in phosphate-buffered saline (PBS). Optical density was measured, and the bacteria samples were adjusted to the desired concentration. The final E. coli preparation contained $10^{7}$ colony forming units (CFUs) in $0.05 \mathrm{~mL}$ PBS [18].

\section{Animal model}

All of the experimental protocols were approved by the Institutional Animal Care and Use Committee of Samsung Biomedical Research Institute. The study followed the institutional and National Institutes of Health guidelines for laboratory animal care.

Eight-week-old male ICR mice were purchased from Orient Co. (Seoul, Korea) and housed in individual cages with free access to water and laboratory chow. Animals were divided into four groups: sham control $(\mathrm{S}, \mathrm{n}=57)$, E. coli-induced ALI control $(\mathrm{E}, \mathrm{n}=125)$, ALI with fibroblast transplantation ( $\mathrm{F}, \mathrm{n}=58)$, and ALI with human UCB-derived MSCs transplantation (M, n = 104).

To induce ALI, mice were anesthetized with an intraperitoneal injection of a mixture of ketamine and xylazine $(45 \mathrm{mg} / \mathrm{kg}$ and $8 \mathrm{mg} / \mathrm{kg}$, respectively). Briefly, each mouse was restrained at a $70^{\circ}$ angle against a plastic wall, an otoscope was employed to visualize the vocal 
cords, and intubation was performed with a 20-gauge central catheter (Leader Cath $20 \mathrm{G} \times 8 \mathrm{~cm}$; Vygon, Paris, France). E. coli at $10^{7}$ CFUs in $0.05 \mathrm{~mL}$ PBS was administered, followed by $2 \mathrm{~cm} \mathrm{H}_{2} \mathrm{O}$-pressure air inflation to ensure an even bacterial distribution. After the E. coli instillation procedure, which was completed within $30 \mathrm{sec}$ for each mouse, the catheters were removed and the animals were allowed to recover and subsequently returned to their dams.

For cell transplantation, $1 \times 10^{5}$ cells (MSCs in $\mathrm{M}$ or fibroblasts in F) in $0.05 \mathrm{~mL}$ PBS were administered intratracheally three hours after the E. coli instillation. Mice in groups $\mathrm{S}$ and $\mathrm{E}$ received the same volume of PBS intratracheally. For intratracheal transplantation, the animals were anesthetized and the catheter was placed as described above. After intratracheal transplantation, the catheter was removed and the mice were allowed to recover and subsequently returned to their dams.

Intraperitoneal antibiotics (ceftriaxone, $100 \mathrm{mg} / \mathrm{kg}$ once a day) were administered for three consecutive days after the injury. Mice were sacrificed at post-injury days 1, 3 and 7, and each animal was allocated to a morphometric or biochemical group. Survival rates were assessed for seven days after injury in the 7-day experimental groups.

\section{Tissue preparation}

Lung tissue was prepared from surviving animals at post-injury days 1, 3 and 7. The mice were anesthetized with sodium pentobarbital (100 $\mathrm{mg} / \mathrm{kg})$, and the lungs and heart were exposed via thoracotomy, followed by transcardiac perfusion with ice-cold PBS.

The lungs were fixed by tracheal instillation of $4 \%$ formaldehyde with a constant inflation pressure of $20 \mathrm{~cm}$ $\mathrm{H}_{2} \mathrm{O}$. The trachea was ligated, and the lungs were removed and immersed in $4 \%$ formaldehyde overnight at room temperature. Both lungs were embedded in paraffin, and transverse serial sections ( $4 \mu \mathrm{m}$ thick) were prepared for morphometric analyses. For the biochemical analyses, the lungs were excised, frozen in liquid nitrogen, and homogenized.

\section{Morphometric analyses}

Four-micrometer-thick sections were stained with hematoxylin and eosin. Two sections per mouse were randomly chosen for the analysis, and three random microscopic fields of the distal lung were evaluated by a blinded observer. Lung injury was scored according to the following four categories: alveolar congestion, hemorrhage, neutrophil infiltration into the airspace or vessel wall, and alveolar wall thickness/hyaline membrane formation. Each category was graded on a five point scale: $0=$ minimal injury, $1=$ injury up to $25 \%$ of the field, $2=$ injury up to $50 \%$ of the field, $3=$ injury up to $75 \%$ of the field, and $4=$ diffuse injury [19].

\section{Myeloperoxidase (MPO) activity assay}

MPO activity in the homogenized lung tissues was measured as described by Gray et al [20]. One unit of MPO activity was defined as the quantity of enzyme needed to degrade $1 \mu \mathrm{mol}$ of peroxide $/ \mathrm{min}$ at $25^{\circ} \mathrm{C}$.

\section{Enzyme-linked immunosorbent assay (ELISA)}

The frozen lungs were homogenized in cold buffer (50 mM Tris- $\mathrm{HCl}, \mathrm{pH} 7.4$ ) with $1 \mathrm{mM}$ EDTA, $1 \mathrm{~mL}$ EGTA, $1 \mathrm{mM}$ PMSF, $42 \mathrm{mM} \mathrm{KCl}$, and $5 \mathrm{mM} \mathrm{MgCl}_{2}$. The samples were centrifuged at $8,000 \mathrm{~g}$ for $20 \mathrm{~min}$ at $4^{\circ} \mathrm{C}$ to remove cellular debris. The protein content in the supernatant was measured using the Bradford method with a bovine serum albumin (Sigma-Aldrich, St. Louis, MO, USA) standard. Lung interleukin (IL)- $1 \alpha$, IL-1 $\beta$, IL-6, tumor necrosis factor (TNF)- $\alpha$, and macrophage inflammatory protein (MIP)-2 levels were measured using the Milliplex MAP ELISA Kit according to the manufacturer's protocol (Millipore, Billerica, MA, USA).

\section{Protein macroarray}

Each lung lysate was analyzed using a mouse cytokine array kit (Proteome Profiler ${ }^{\mathrm{TM}}$; R\&D Systems, Minneapolis, $\mathrm{MN}, \mathrm{USA}$ ). A total of $250 \mu \mathrm{g}$ of lysate was incubated in the nitrocellulose membrane array overnight at $4^{\circ} \mathrm{C}$. After washing away the unbound protein, the array was incubated with a cocktail of phospho-site-specific biotinylated antibodies for $2 \mathrm{~h}$ at room temperature, followed by streptavidin-HRP for $30 \mathrm{~min}$. Signals were visualized with chemiluminescent reagents (Amersham Biosciences, Pittsburgh, PA, USA), and recorded on X-ray film. The arrays were scanned, and optical densities were measured using Image J software (NIH) and compared among the experimental groups.

The protein macroarray analysis included inflammatory cytokines of interest, including complement $5 \mathrm{a}$ (C5a), the soluble form of intercellular adhesion molecule (sICAM)1, IL-1 $\alpha$, IL-1 $\beta$, IL-6, IL-16, interferon-inducible protein (IP)-10, the murine analogue of monocyte chemoattractant protein (MCP)-1 (JE), MCP-5, MIP- $1 \alpha$, MIP- $1 \beta$, MIP-2, regulated upon activation normal T-expressed and presumably secreted (RANTES), tissue inhibitor of metalloproteinase (TIMP)-1, TNF- $\alpha$, and triggering receptor expressed on myeloid cells (TREM)-1.

\section{Wet-dry lung ratios}

The lungs from each animal were removed, placed into a microtube, and weighed. The lungs were then dried at $60^{\circ} \mathrm{C}$ for $72 \mathrm{~h}$ and weighed again. The wet lung mass divided by the dry lung mass represented the wet-dry 
lung ratio, which indicates the fraction of wet lung weight due to water.

\section{Bacterial quantification}

Bacterial concentrations were measured in BAL fluid and blood from the E, F, and M groups at post-injury days 1, 3 and 7. Mice were anesthetized, thoracotomy was performed as described above, and intracardiac blood was obtained aseptically, followed by transcardiac perfusion with PBS. BAL fluids were obtained via an aseptic saline irrigation.

Bacteria CFU levels in BAL fluids and blood were measured at dilutions of $10^{-3} \sim 10^{-6}$ plated on BHI agar after overnight incubation at $37^{\circ} \mathrm{C}$.

Assessment of direct inhibition of bacterial growth by MSCs or fibroblast was done by counting CFUs in vitro. Dulbecco's modified Eagle's medium (DMEM) was used as a control medium and human fibroblasts (MRC-5; Korean Cell Line Bank No.10171) were used as a control cells. In brief, each 3 well-plates of DMEM, fibroblast, and MSCs in 12 -well plates $\left(10^{5}\right.$ cells per well) in BHI media were infected with $10^{3}$ CFUs $E$. coli and incubated for 6 hours in humidified $\mathrm{CO}_{2}$ incubator, then aliquots of culture medium were taken from each well, serially diluted with sterile PBS, and plated on agar plates. Colonies were counted after overnight incubation at $37^{\circ} \mathrm{C}$.

\section{Statistical analyses}

The data are expressed as the mean \pm SEM. Survival rates were compared using the Kaplan-Meier analysis followed by a $\log$ rank test. For continuous variables with a normal distribution, the groups were compared using a $t$-test with a Bonferroni correction. Continuous variables that were not normally distributed were analyzed using the Wilcoxon rank test with a Bonferroni correction. All data were analyzed using SPSS version 12.0 (SPSS Inc., Chicago, IL, USA). Values of $p<0.05$ were considered statistically significant.

\section{Results}

\section{Survival rate}

Despite antibiotic treatment, E. coli-induced ALI (E) significantly reduced the survival rate $(79 \%, 42 / 53$ mice surviving) at post-injury day 7 compared to the $100 \%$ survival rate $(22 / 22)$ of the sham control group $(S)$. The reduced survival rate observed in $\mathrm{E}(p<0.05$ vs. S) improved with MSC treatment (M) (95\%, 36/38 mice surviving; $p>0.05$ vs. $\mathrm{S}$ and $p<0.05$ vs. E), but not with fibroblast treatment (F) $(p<0.05$ vs. S, $p=0.91$ vs. E) (Figure 1).

\section{Lung histology, injury scores, and MPO activity}

Representative photomicrographic lung histology in group $\mathrm{E}$ showed increased congestion and cellular

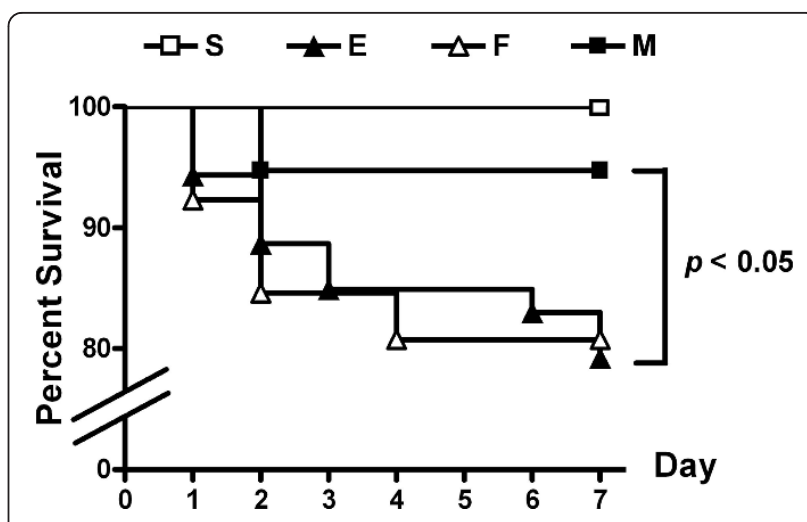

Figure 1 Survival Rates. Survival rates determined using the Kaplan-Meier analysis followed by a log rank test. S, sham group; $E_{\text {, }}$ E. coli-induced ALI control group; F, fibroblast transplant ALI group; M, human UCB-derived MSC transplant ALI group.

infiltration at post-injury days 1,3 and 7 , with peak inflammatory activity observed at day 3 compared with that of S (Figure 2). To quantify the differences, lung injury was scored according to the degree of alveolar congestion, hemorrhage, neutrophil infiltration, and wall thickening (Figure 3).

Group E showed significant increases in all injury scores at post-injury day 1 ( $p<0.01$ vs. S for alveolar congestion and leukocyte infiltration; $p<0.05$ vs. S for alveolar hemorrhage and wall thickening) and day $3(p<0.01$ vs. $\mathrm{S}$ for all scores) but not at day 7 ( $p>0.05$ vs. $\mathrm{S}$ in all scores). MSC transplantation (M) significantly attenuated the ALIinduced increases in injury scores at post-injury day $3(p<$ 0.01 vs. E for alveolar congestion and leukocyte infiltration; $p<0.05$ vs. E for alveolar wall thickening), except for alveolar hemorrhage ( $p>0.05$ vs. E), while fibroblast transplantation (F) did not attenuate and even aggravated the injury scores for alveolar hemorrhage and leukocyte infiltration ( $p<0.01$ vs. E) at post-injury day 3 .

MSC transplantation (M, $p<0.01$ vs. E), but not fibroblast transplantation (F, $p>0.05$ vs. E), significantly attenuated ALI-induced increases in lung MPO activity (an indication of neutrophil accumulation) (E, $p<0.01$ vs. S) at post-injury day 3 (Figure 4).

\section{Proinflammatory cytokines}

The protein levels of IL- $1 \alpha$, IL- $1 \beta$, IL- 6 , TNF- $\alpha$, and MIP-2 measured by ELISA in lung homogenates were significantly higher in group $\mathrm{E}$ compared to $\mathrm{S}$ at postinjury days 1 and 3 (E, $p<0.01$ vs. $S$ for all), and to a lesser extent at post-injury day 7 (E, $p<0.01$ vs. $S$ for IL- $1 \alpha$, IL- $1 \beta$, TNF- $\alpha$, and MIP-2; $p<0.01$ vs. S for IL-6). This ALI-induced increase in cytokine levels was significantly attenuated by MSC transplantation (M) but not by fibroblast transplantation (F) at post-injury days $3(\mathrm{M}, p<$ 0.01 vs. E), and 7 (M, $p>0.05$ vs. S) (Figure 5). 

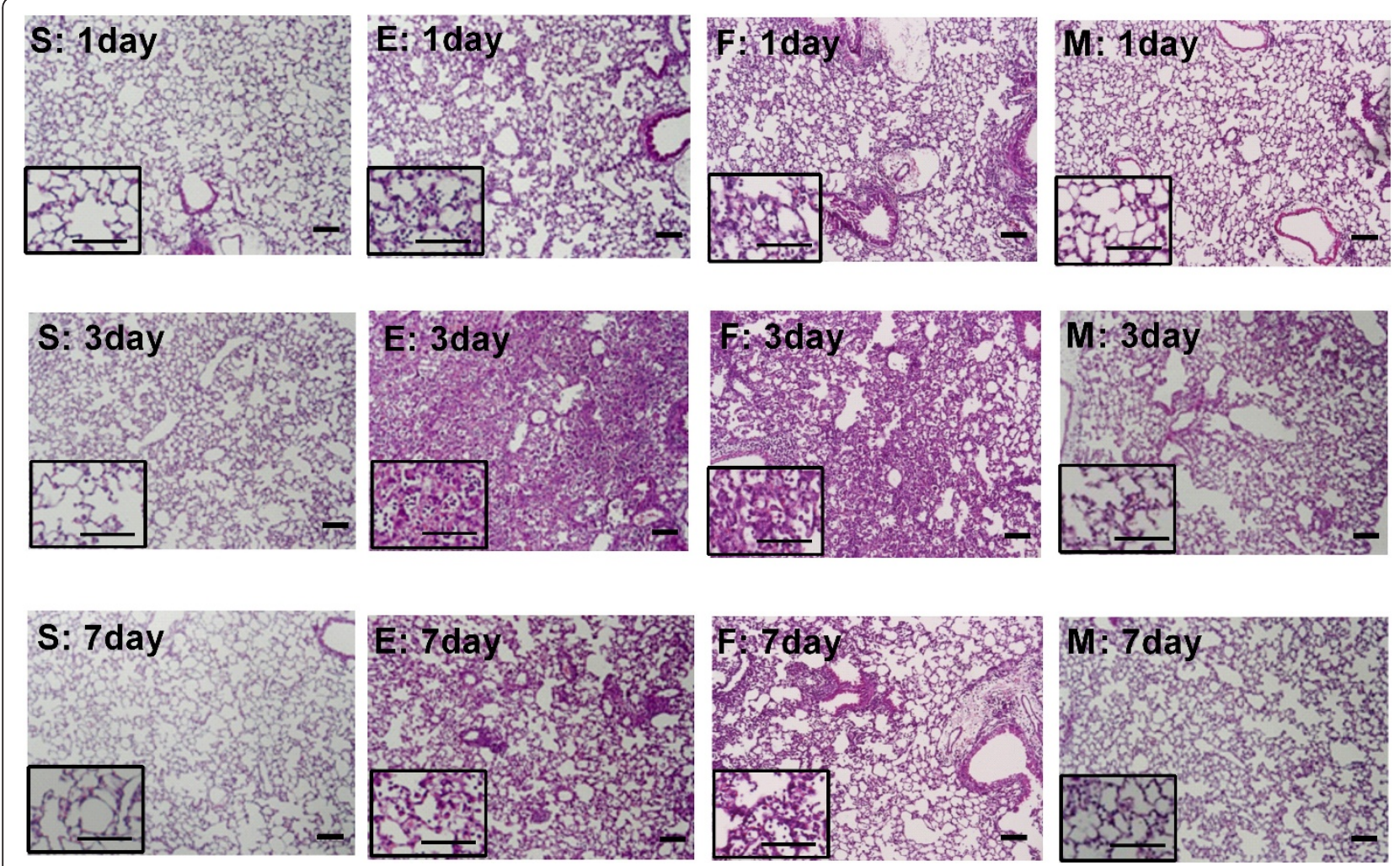

Figure 2 Gross Histologic Evaluation. Photomicrographs of hematoxylin and eosin staining of each group at three time points. (Scale bar $=25$ $\mu \mathrm{m}$ and $10 \mu \mathrm{m}$ in the magnified photograph).

\section{Lung cytokine profiles}

Lung homogenates at post-injury day 3 were used for a mouse macroarray experiment to profile various inflammatory cytokine proteins involved in the peak inflammatory reactions induced by ALI with $E$. coli instillation. Protein levels of inflammatory cytokines from lung homogenates, including C5a, sICAM-1, IL- $1 \alpha$, IL-1 $\beta$, IL-6, IL-16, IP-10, JE, MCP-5, MIP-1 $\alpha$, MIP-1 $\beta$, MIP-2, RANTES, TIMP-1, TNF- $\alpha$, and TREM-1, were significantly elevated in $\mathrm{E}(p<0.01$ vs. S, except for TNF- $\alpha$ : $p$ $<0.05$ vs. S). MSC transplantation reduced the levels of C5a, MIP-2, RANTES, and TNF- $\alpha$ to S levels (M, $p>$ 0.05 vs. S), markedly reduced the levels of IL-16, MCP5 , MIP- $1 \alpha$, MIP-1 $\beta$, and TREM- 1 (M, $p<0.01$ vs. E), and significantly reduced the levels of IL-1 $\alpha$, IL-1 IL-6, IP-10, and JE ( $p<0.05$ vs. E). Fibroblast transplantation did not attenuate this increase for any cytokine (F, $p<0.01$ vs. S; $p>0.05$ vs. E) (Figure 6).

\section{Lung water content}

E. coli-induced ALI (E) increased lung water content expressed as the wet-dry lung ratio, which suggested increased permeability compared $\mathrm{S}$ at post-injury. The increase in lung water content was marked at day 3 (E, $p<0.01$ vs. S) and to a lesser extent at day 7 (E, $p<$
0.05 vs. S). MSCs transplantation (M, $p<0.05$ vs. E) but not fibroblast transplantation (F, $p>0.05$ vs. E) reduced lung water content significantly at post-injury day 3 (Figure 7).

\section{Bacterial counts}

To evaluate the bacterial burdens, the number of colony forming units (CFUs) was counted in BAL fluids and blood from animals in the E, F, and M groups at postinjury days 1,3 , and 7 . Bacterial counts in both BAL and blood were significantly lower in $\mathrm{M}$ compared to $\mathrm{E}$ at post-injury day 7 (M, $p<0.01$ vs. E); however, bacterial counts did not different between $\mathrm{F}$ and $\mathrm{E}$ at postinjury day 7 (Figure 8A-B).

Bacterial growth depending on the presence of MSCs was evaluated in vitro. Six hours after incubation, bacterial growth was significantly inhibited by MSC (M, $p<$ 0.01 vs. $\mathrm{E}$ and $\mathrm{F}$ ), but not by fibroblast (F, $p>0.05$ vs. E) (Figure 8-C).

\section{Discussion}

A major risk of ALI/ARDS is infection, such as severe sepsis or pneumonia, which causes most cases of mortality [2-4,21]. Lipopolysaccharide (LPS)-induced lung injury has been used as an animal model to mimic 


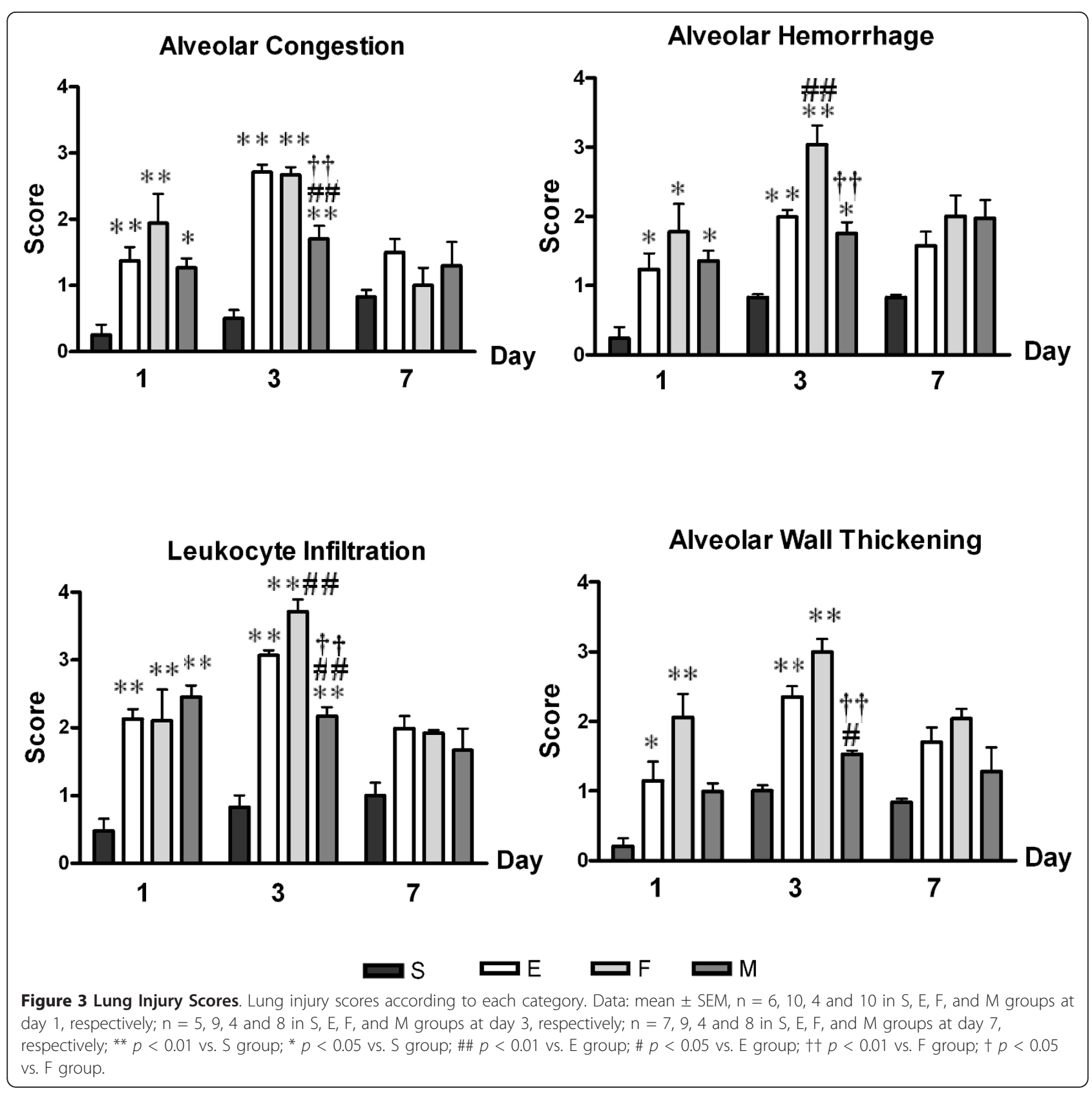

infectious human ALI and to stimulate host inflammatory responses [19,22-24]. However, LPS-induced ALI causes endothelial or epithelial injury that is less severe than that seen in human ALI [22]. In contrast, instillation of $E$. coli into mouse lungs resulted in ALI with a presentation similar to that of human ALI, including severe pneumonia and sepsis resulting in bilateral lung edema, alveolar hemorrhage, leukocyte infiltration, and alveolar wall thickening with severe inflammatory responses. Severe inflammatory responses were evidenced by lung MPO activity that was 20-fold greater than that of LPSinduced lung injury [25], a surge of inflammatory cytokines in lung homogenates, and increased water content in the lungs corresponding to an increased wet-dry lung ratio that peaked at post-injury day 3. Furthermore, the mortality rate in $E$. coli-induced ALI mice was about $30 \%$ with antibiotics treatment, which is consistent with infection related-ARDS mortality in clinical settings [4]. Overall, these findings suggest that $E$. coli-induced ALI resulting in severe inflammation is the most clinically relevant model to date.

In this study, intratracheal transplantation of human UCB-derived MSCs into E. coli-induced ALI mice significantly increased survival and attenuated lung injuries. 


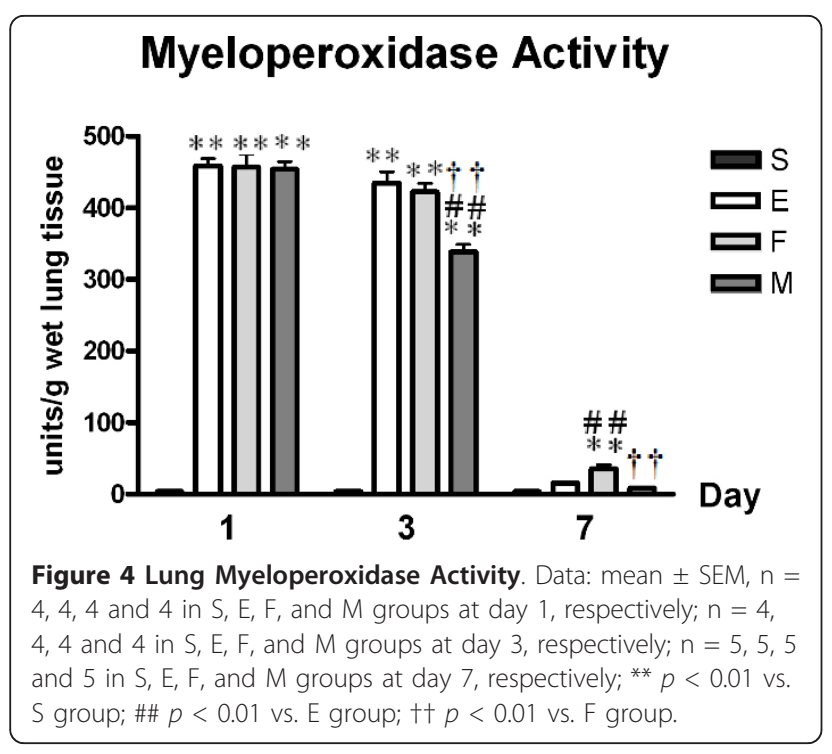

The protective effect of MSCs was evidenced by decreased injury scores at post-injury day 3 and attenuated lung inflammation, including a reduction in MPO activity and inflammatory cytokine protein levels, at postinjury days 3 and 7. MSC transplantation also reduced the elevated lung water content at post-injury day 3 and bacterial counts in blood and BAL fluid at post-injury day 7 in vivo as well as in vitro. Thus, our results support the potential use of human UCB-derived MSCs as a new therapeutic modality for $E$. coli-induced ALI.

Although the mechanism underlying the therapeutic effect of MSCs on ALI has yet to be elucidated, the anti-inflammatory properties of MSCs may contribute to their protective role in ALI $[7,8]$. Recent ALI studies using BM-MSCs have also suggested their anti-inflammatory effects as the main protective mechanism $[9,19,26,27]$. Moreover, in previous studies, we successfully xenotransplanted human UCB-derived MSCs into

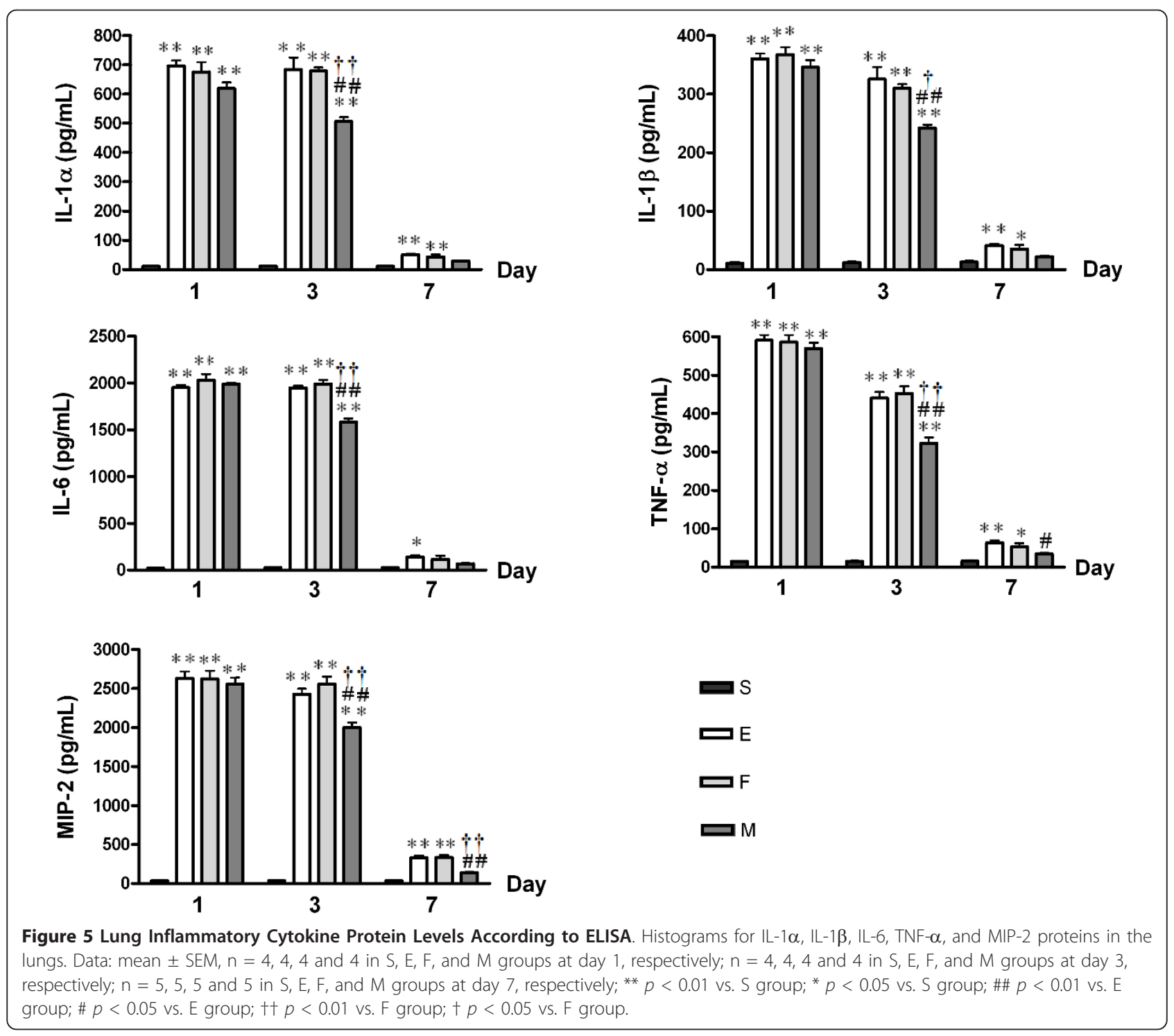




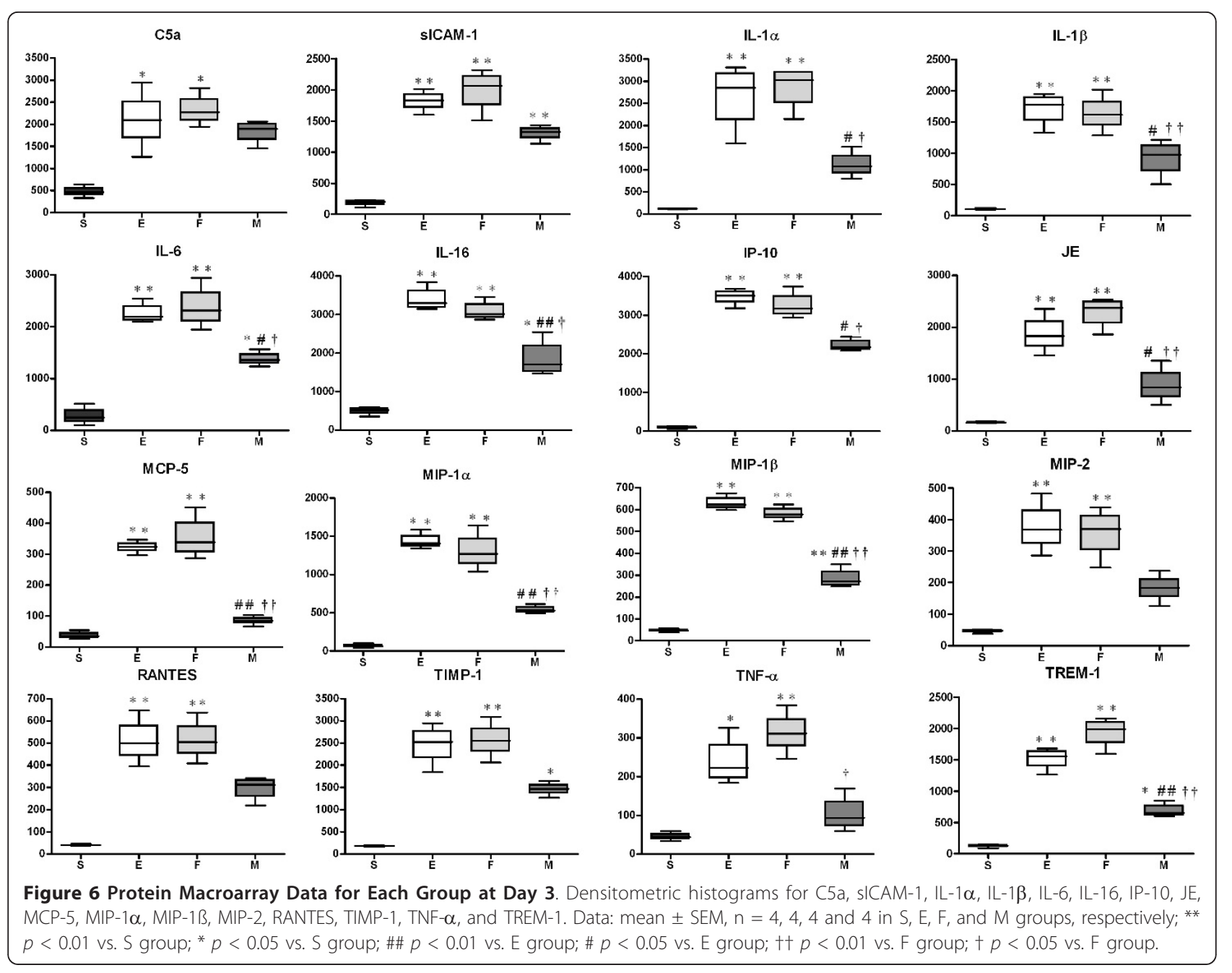

immunocompetent newborn rats and demonstrated their efficacy in reducing hyperoxic lung injury, mainly through anti-inflammatory effects $[14,15]$. Here, we demonstrate attenuated lung MPO activity, which is an indicator of neutrophil accumulation or activity, resulting from intratracheal human UCB-derived MSC transplantation into ALI mice. The study also investigated inflammatory cytokine levels from lung homogenates as possible direct markers of lung inflammation. Protein levels of pro-inflammatory cytokines including IL-1 $\alpha$, IL- $1 \beta$, IL-6, TNF- $\alpha$, and MIP-2 were consistently reduced by MSC transplantation in ALI mice. These results clearly indicate an association between the attenuation caused by human UCB-derived MSCs in $E$. coli-induced ALI and these anti-inflammatory effects of these cells.

We used a protein macroarray to analyze lung inflammatory cytokines to elucidate the possible mechanism underlying the down-modulation of inflammation by MSCs in ALI mice. MSC transplantation showed suppressed pro-inflammatory molecules (IL-1 $\alpha$, IL-1 $\beta$, IL-6, TNF- $\alpha$ ), down-modulated the chemotactic effects of neutrophils, immature dendrocytes, and natural killer cells (MIP- $1 \alpha$, MIP-1 $\beta$, RANTES), and decreased the chemotactic effects of T-cells (IP-10). These results indicate an overall decrease in Toll-like receptor (TLR) signaling [28-30]. TLRs are common immune molecules that recognize bacterial pathogens in acute lower respiratory bacterial infections [28]. Moreover, TLRs are pattern recognition receptors that control lung homeostasis and play a key role in both infectious and sterile lung inflammation [31]. Thus, human UCB-derived MSCs may modulate TLR signaling to attenuate the inflammation caused by $E$. coli-induced ALI. Another innate immune protein class that can either amplify or dampen TLR-induced signals is triggering receptor expressed on myeloid cells (TREM) [32]. TREM-1 is a well-known inflammatory amplifier [31]; thus, MSCs might also modulate the TREM-1 pathway that cross-reacts with TLR signaling. Other lung proteins attenuated by human UCB-derived 


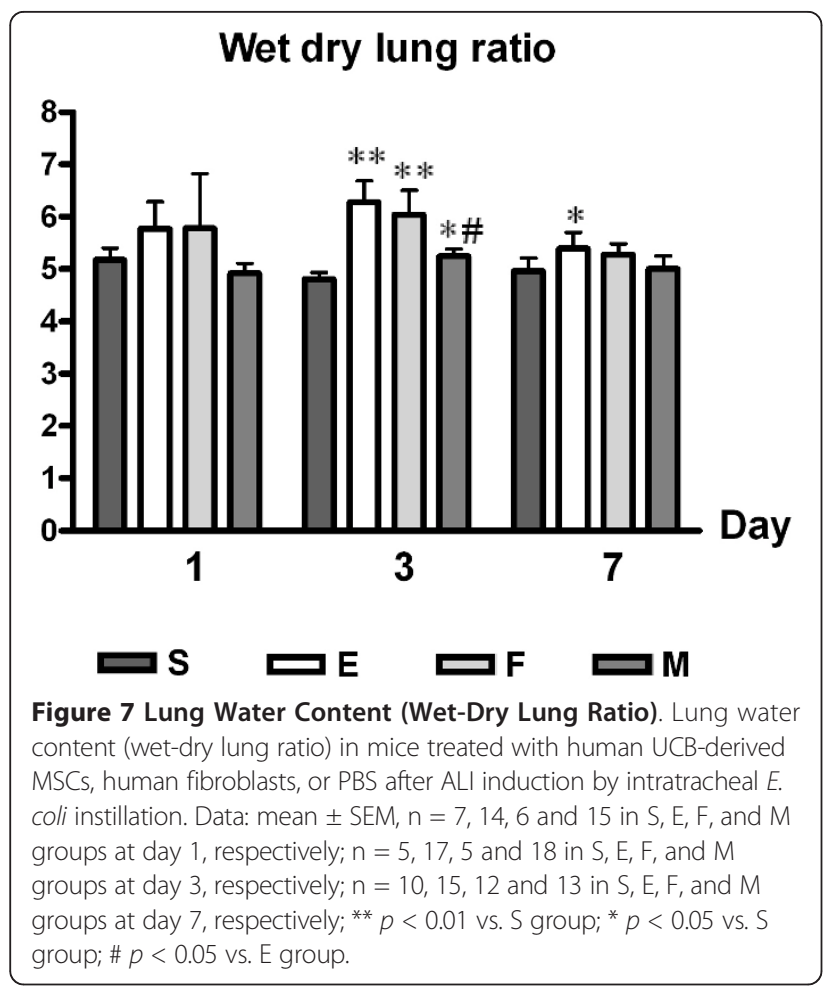

MSCs, such as MCP-1 (JE), MCP-5, IL-16, and TIPM-1, participate in inflammatory processes by $\mathrm{T}$ cell activation and cell migration. Although the proteins presented in this study do not form a clear pathway network, downregulation of $E$. coli-induced ALI by intratracheal transplantation of human UCB-derived MSCs was clearly associated with the control of complex inflammatory interactions.

Human UCB-derived MSCs seem to have an impact on endothelial and epithelial homeostasis in ALI. The increased wet-dry lung ratio in ALI implies lung edema which is primarily due to disrupted alveolar barrier integrity maintained by both the lung endothelium and epithelium. In this study, treatment with human UCB-derived MSCs improved the wet-dry lung ratio in ALI mice, suggesting that UCB-derived MSCs might have a role in repairing alveolar barrier integrity. The epithelial sodium channel $(\mathrm{ENaC})$ and $\mathrm{Na}-\mathrm{K}$ ATPase in type II pneumocytes have been postulated as candidate sites important for modulation by MSCs [33], probably by paracrine effects such as keratinocyte growth factor (KGF) [23]. Endothelial permeability modulation may be another mechanism of action of MSCs, and KGF and angiopoietin-1 are

(A) BAL Fluid Cultures

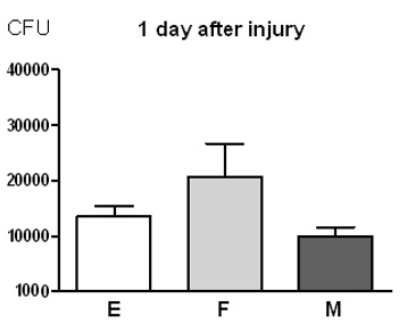

(B) Blood Cultures

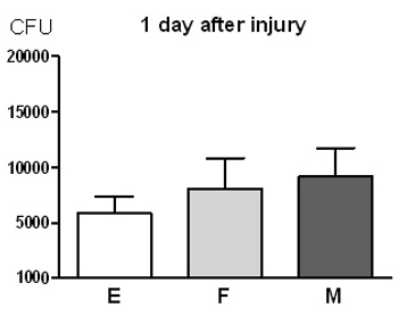

(C) In Vitro Cultures

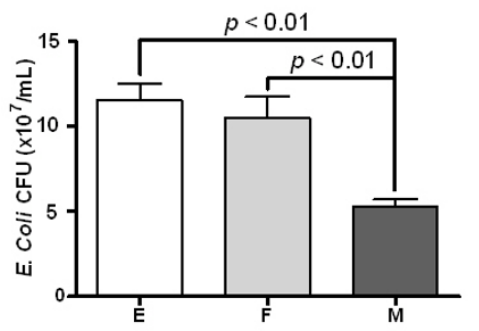

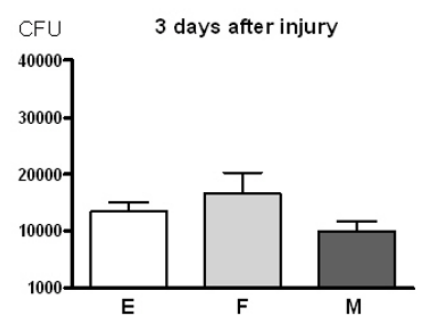
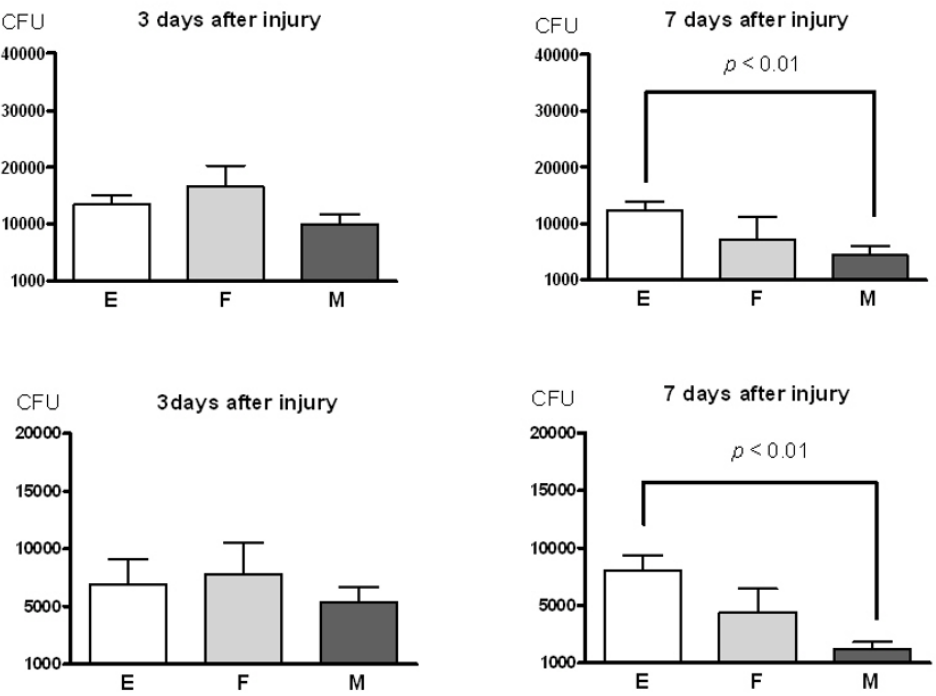

Figure 8 Bacterial Growth in vivo (A and B) and in vitro (C). Bacterial colony forming units in BAL fluid (A) and blood (B) at 1, 3, and 7 days after injury in the $E, F$, and $M$ groups. Data: mean \pm SEM. ( $n=10,5$, and 10 in the $E, F$, and $M$ groups, respectively). (C) Bacterial colony forming units in media after $6 \mathrm{~h}$ incubation of $E$. coli with culture media only (E), fibroblasts (F), and human UCB-derived MSCs (M). Data: mean \pm SEM. ( $n=8,8$, and 8 in the $E, F$, and $M$ groups, respectively). 
potentially important molecules involved in this effect [7,34-36]. Although MSCs have been shown to uniformly improve wet-dry lung ratios in ALI models [23,24,26], the precise action of MSCs on alveolar barrier integrity needs to be further investigated.

In this study, human UCB-derived MSC transplantation reduced bacterial concentration in bronchoalveolar spaces and blood of E. coli-induced ALI mice, and in vitro data also support an inhibiting effect of bacterial growth by human UCB-derived MSCs, not by fibroblasts, which is consistent with the study by Mei et al. in a sepsis model [9]. Recently, a study using BM-MSCs in a bacteriainduced ALI model suggested a paracrine effect by an antibacterial peptide from MSCs [10]. Because inflammatory cells contain many antibacterial peptides, MSCs might augment the antibacterial activity of those cells or secrete antibacterial peptides directly. Because little is known about the antibacterial activities of MSCs, additional in vivo and in vitro studies should be conducted to confirm the utility of MSCs in the treatment of bacterial diseases.

As a limitation of the study, we performed tissue examinations only in surviving animals at post-injury days 1,3 , and 7 . Thus, this method may contribute to the lack of a difference at day 7 , possibly resulting in an under-estimation of the therapeutic effect of MSCs.

Nevertheless, this study is unique in establishing the E. coli induced acute lung injury animal model and testing the protective anti-inflammatory and bactericidal effects of local intra-tracheal xeno-transplantation of human UCB derived MSCs . E. coli induced ALI animal model is essential for further studies to elucidate the mechanism of protective anti-inflammatory and bactericidal effects of human UCB derived MSCs observed in the present study in the near future.

\section{Conclusions}

This study suggests that intratracheal transplantation of human UCB-derived MSCs into mice with E. coliinduced ALI significantly improves survival and attenuates ALI, primarily through anti-inflammatory mechanisms. Moreover, it is thought that human UCB-derived MSCs might have additional beneficial effects on E. coliinduced ALI, such as alveolar epithelial barrier repair and bacterial clearance. UCB is a clinically promising source of MSCs and our findings suggest human UCBderived MSCs transplantation as a new therapeutic modality for reducing the high mortality and morbidity of human ALI.

\section{List of abbreviations}

ALI: acute lung injury; ARDS: acute respiratory distress syndrome; BAL: bronchoalveolar lavage; E. coli: Escherichia coli; MPO: myeloperoxidase; MSC: mesenchymal stem cell; UCB: umbilical cord blood

\section{Acknowledgements}

This work was supported by a grant from the Korean Healthcare Technology R\&D Project, Ministry for Health, Welfare \& Family Affairs, Republic of Korea (A100361), by the IN-SUNG Foundation of Medical Research (CB08141), and by a Samsung Biomedical Research Institute Grant (\#SBRI CB02211).

\section{Author details}

${ }^{1}$ Department of Pediatrics, Samsung Medical Center, Sungkyunkwan University School of Medicine, Seoul, Korea. ${ }^{2}$ Samsung Biomedical Research Institute, Samsung Medical Center, Seoul, Korea. ${ }^{3}$ Biomedical Research Institute, MEDIPOST Co., Ltd., Seoul, Korea.

\section{Authors' contributions}

ESK: performed animal experiments, the statistical analyses, and wrote the manuscript.

YSC: supervised the study and participated in analyses and writing.

SJC: involved in study design and prepared MSCS.

JKK: performed animal experiments and the statistical analyses.

HSY: performed animal experiments and the statistical analyses.

SYA: performed animal experiments and the statistical analyses.

DKS: performed ELISA and macroarray analysis

SYK: performed animal experiments, histology and scoring, analyzed the results.

YRP: carried out animal experiments.

WSP: conceived, supervised and coordinated the study.

All authors contributed to the analyses and interpretation of the data. All authors have read and approved the final manuscript.

\section{Competing interests}

The authors declare that they have no competing interests.

Received: 30 April 2011 Accepted: 15 August 2011

Published: 15 August 2011

\section{References}

1. Ware LB, Matthay MA: The acute respiratory distress syndrome. N Engl J Med 2000, 342:1334-1349.

2. Rubenfeld GD, Caldwell E, Peabody E, Weaver J, Martin DP, Neff M, Stern EJ, Hudson LD: Incidence and outcomes of acute lung injury. $N$ Engl J Med 2005, 353:1685-1693.

3. Sheu CC, Gong MN, Zhai R, Chen F, Bajwa EK, Clardy PF, Gallagher DC, Thompson BT, Christiani DC: Clinical characteristics and outcomes of sepsis-related vs non-sepsis-related ARDS. Chest 2010, 138:449-467.

4. Hu X, Qian S, Xu F, Huang B, Zhou D, Wang Y, Li C, Fan X, Lu Z, Sun B: Incidence, management and mortality of acute hypoxemic respiratory failure and acute respiratory distress syndrome from a prospective study of Chinese paediatric intensive care network. Acta Paediatr 2010, 99:715-721.

5. Ware LB, Koyama T, Billheimer DD, Wu W, Bernard GR, Thompson BT, Brower RG, Standiford TJ, Martin TR, Matthay MA: Prognostic and pathogenetic value of combining clinical and biochemical indices in patients with acute lung injury. Chest 2010, 137:288-296.

6. Cribbs SK, Matthay MA, Martin GS: Stem cells in sepsis and acute lung injury. Crit Care Med 2010, 38:2379-2385.

7. Lee JW, Gupta N, Serikov V, Matthay MA: Potential application of mesenchymal stem cells in acute lung injury. Expert Opin Blol Ther 2009, 9:1259-1270.

8. Matthay MA, Goolaerts A, Howard JP, Lee JW: Mesenchymal stem cells for acute lung injury: preclinical evidence. Crit Care Med 2010, 38:S569-573.

9. Mei SH, Haitsma JJ, Dos Santos CC, Deng Y, Lai PF, Slutsky AS, Liles WC, Stewart DJ: Mesenchymal stem cells reduce inflammation while enhancing bacterial clearance and improving survival in sepsis. Am J Respir Crit Care Med 2010, 182:1047-1057.

10. Krasnodembskaya A, Song Y, Fang X, Gupta N, Serikov V, Lee JW, Matthay MA: Antibacterial effect of human mesenchymal stem cells is mediated in part from secretion of the antimicrobial peptide LL-37. Stem Cells 2010, 29:2229-2238.

11. Rocha V, Wagner JE Jr, Sobocinski KA, Klein JP, Zhang MJ, Horowitz MM, Gluckman E: Graft-versus-host disease in children who have received a cord-blood or bone marrow transplant from an HLA-identical sibling. Eurocord and International Bone Marrow Transplant Registry Working 
Committee on Alternative Donor and Stem Cell Resources. N Engl J Med 2000, 342:1846-1854.

12. Le Blanc K, Tammik C, Rosendahl K, Zetterberg E, Ringdén O: HLA expression and immunologic properties of differentiated and undifferentiated mesenchymal stem cells. Exp Hematol 2003, 31:890-896.

13. Tse WT, Pendleton JD, Beyer WM, Egalka MC, Guinan EC: Suppression of allogeneic T-cell proliferation by human marrow stromal cells: Implications in transplantation. Transplantation 2003, 75:389-397.

14. Chang YS, Oh W, Choi SJ, Sung DK, Kim SY, Choi EY, Kang S, Jin HJ, Yang YS, Park WS: Human umbilical cord blood-derived mesenchymal stem cells attenuate hyperoxia-induced lung injury in neonatal rats. Cell Transplant 2009, 18:869-886.

15. Chang YS, Choi SJ, Sung DK, Kim SY, Oh W, Yang YS, Park WS: Intratracheal transplantation of human umbilical cord blood derived mesenchymal stem cells dose-dependently attenuates hyperoxia-induced lung injury in neonatal rats. Cell Transplant 2011.

16. Cordero L, Rau R, Taylor D, Ayers LW: Enteric gram-negative bacilli bloodstream infections: 17 years' experience in a neonatal intensive care unit. Am J Infect Control 2004, 32:189-195.

17. Park WS, Chang YS, Lee M: N(omega) -nitro-L-arginine methyl ester (L-NAME) attenuates the acute inflammatory responses and brain injury during the early phase of experimental Escherichia coli meningitis in the newborn piglet. Neurol Res 2001, 23:862-868.

18. Serikov VB, Mikhaylov VM, Krasnodembskay AD, Matthay MA: Bone marrow-derived cells participate in stromal remodeling of the lung following acute bacterial pneumonia in mice. Lung 2008, 186:179-190.

19. Gupta N, Su X, Popov B, Lee JW, Serikov V, Matthay MA: Intrapulmonary delivery of bone marrow-derived mesenchymal stem cells improves survival and attenuates endotoxin-induced acute lung injury in mice. J Immunol 2007, 179:1855-1863.

20. Gray KD, Simovic MO, Chapman WC, Blackwell TS, Christman JW, May AK, Parman KS, Stain SC: Endotoxin potentiates lung injury in ceruleininduced pancreatitis. Am J Surg 2003, 186:526-530.

21. Zimmerman JJ, Akhtar SR, Caldwell E, Rubenfeld GD: Incidence and outcome of pediatric acute lung injury. Pediatrics 2009, 124:87-95.

22. Matute-Bello G, Frevert CW, Martin TR: Animal models of acute lung injury. Am J Physiol Lung Cell Mol Physiol 2008, 295:L379-399.

23. Lee JW, Fang X, Gupta N, Serikov V, Matthay MA: Allogeneic human mesenchymal stem cells for treatment of E. coli endotoxin-induced acute lung injury in the ex vivo perfused human lung. Proc Natl Acad Sci USA 2009, 106:16357-16362.

24. Mao M, Wang SN, Lv XJ, Wang Y, Xu JC: Intravenous delivery of bone marrow-derived endothelial progenitor cells improves survival and attenuates lipopolysaccharide-induced lung injury in rats. Shock 2010, 34:196-204.

25. Kabir K, Gelinas JP, Chen M, Chen D, Zhang D, Luo X, Yang JH, Carter D, Rabinovici R: Characterization of a murine model of endotoxin-induced acute lung injury. Shock 2002, 17:300-303.

26. Xu J, Woods CR, Mora AL, Joodi R, Brigham KL, lyer S, Rojas M: Prevention of endotoxin-induced systemic response by bone marrow-derived mesenchymal stem cells in mice. Am J Physiol Lung Cell Mol Physiol 2007, 293:L131-141

27. Rojas M, XU J, Woods CR, Mora AL, Spears W, Roman J, Brigham KL: Bone marrow-derived mesenchymal stem cells in repair of the injured lung. Am J Respir Cell Mol Biol 2005, 33:145-152.

28. Balamayooran G, Batra S, Fessier MB, Happel KI, Jeyaseelan S: Mechanisms of neutrophil accumulation in the lungs against bacteria. Am J Respir Cell Mol Biol 2010, 43:5-16.

29. Mitchell D, Olive D: Regulation of Toll-like receptor-induced chemokine production in murine dendritic cells by mitogen-activated protein kinases. Mol Immunol 2010, 47:2065-2073.

30. Qian C, An H, Yu Y, Liu S, Cao X: TLR agonists induce regulatory dendritic cells to recruit Th1 cells via preferential IP-10 secretion and inhibit Th1 proliferation. Blood 2007, 109:3308-3315.

31. Opitz B, van Laak V, Eitel J, Suttorp N: Innate immune recognition in infectious and noninfectious diseases of the lung. Am J Respir Crit Care Med 2010, 181:1294-1309.

32. Ford JW, McVicar DW: TREM and TREM-like receptors in inflammation and disease. Curr Opin Immunol 2009, 21:38-46.

33. Liu KD, Matthay MA: Advances in critical care for the nephrologist: acute lung injury/ARDS. Clin J Am Soc Nephrol 2008, 3:578-586.
34. Fang $X$, Neyrinck AP, Matthay MA, Lee JW: Allogeneic human mesenchymal stem cells restore epithelial protein permeability in cultured human alveolar type II cells by secretion of angiopoietin-1. J Biol Chem 2010, 285:26211-26222.

35. Xu J, Qu J, Cao L, Sai Y, Chen C, He L, Yu L: Mesenchymal stem cell-based angiotension-1 gene therapy for acute lung injury induced by lipopolysaccharide in mice. J Pathol 2008, 214:472-481.

36. Mei H, McCarter SD, Deng Y, Parker CH, Liles WC, Stewart DJ: Prevention of LPS-induced acute lung injury in mice by mesenchymal stem cells overexpressing angiopoietin 1. PLoS Med 2007, 4:e269.

doi:10.1186/1465-9921-12-108

Cite this article as: Kim et al.: Intratracheal transplantation of human umbilical cord blood-derived mesenchymal stem cells attenuates Escherichia coli-induced acute lung injury in mice. Respiratory Research 2011 12:108.

\section{Submit your next manuscript to BioMed Central and take full advantage of:}

- Convenient online submission

- Thorough peer review

- No space constraints or color figure charges

- Immediate publication on acceptance

- Inclusion in PubMed, CAS, Scopus and Google Scholar

- Research which is freely available for redistribution 